\section{Stephen J. Pennycook to receive MRS Innovation in Materials Characterization Award}

Otephen J. Pennycook, a Corpo$\checkmark$ rate Fellow of Oak Ridge National Laboratory (ORNL) with a joint faculty appointment at the University of Tennessee-Knoxville, has been honored with the Materials Research Society Innovation in Materials Characterization Award for his "pioneering applications of aberration-corrected Z-contrast scanning transmission electron microscopy in the characterization of materials at the atomic scale." He will present an award talk at the 2012 Materials Research Society Spring Meeting in San Francisco. The award has been endowed by Toh-Ming Lu and Gwo-Ching Wang.

In 1992, Pennycook received the MRS Medal for his development of $Z$-contrast imaging in the scanning transmission electron microscope (STEM) which enabled direct determination of the atomicscale structure and chemistry of materials. Pennycook has since pioneered the rapid expansion of aberration-corrected STEM to materials research from catalysis to structural materials, spintronics and optoelectronics, and nanoscience.

In the area of superconductivity, for example, where it had been known that Ca doping improved the grain-boundary critical current in YBCO, the mechanism was thought to involve substitution of $\mathrm{Ca}$ for $\mathrm{Y}$ at the boundary. However, TEM imaging and spectroscopy showed that Ca occupies a variety of sites around the boundary, normally associated with Y, $\mathrm{Ba}$, or $\mathrm{Cu}$ solute. Theory showed this reduced the strain, and the strain reduc- tion allowed $\mathrm{O}$ to return, improving the performance.

More recently, Pennycook and his colleagues identified MRS individual light atoms in monolayer boron nitride $(\mathrm{BN})$ and graphene. In an example where they imaged $\mathrm{C}$ and $\mathrm{O}$ impurities in monolayer $\mathrm{BN}$, they showed that the $\mathrm{O}$ atoms always occupy the $\mathrm{N}$ sites, whereas the $\mathrm{C}$ atoms always substitute for a BN pair. Pennycook and his group demonstrated that annular darkfield imaging in an aberration-corrected STEM optimized for low voltage operation can resolve and identify all individual atoms in non-periodic solids, advancing the field of materials analysis.

Pennycook holds BA, MA, and $\mathrm{PhD}$ degrees from the University of Cambridge, UK. He recently became Fellow of MRS and was awarded the Thomas Young Medal of the Institute of Physics, and the Hsun Lee Award of the Chinese Academy of Sciences. He is co-editor of the book Scanning Transmission Electron Microscopy: Imaging and Analysis (Springer, 2011) and served as coGuest Editor of the January 2012 issue of MRS Bulletin. He has over 350 journal publications, 31 book chapters, three encyclopedia articles, and one patent.

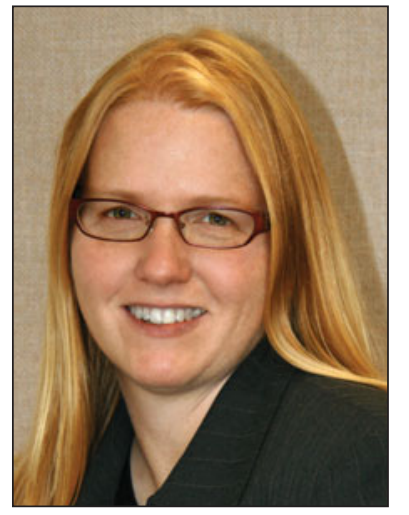

Kristi S. Anseth receives inqugural Mid-Career Researcher Award for biomaterials

T he Materials Research Society has named Tisone Professor, Associate Professor of Surgery, and Howard Hughes Medical Institute Investigator Kristi S. Anseth of the University of Colorado-Boulder to receive the inaugural Mid-Career Researcher Award for her "exceptional achievement at the interface of materials and biology enabling new, functional biomaterials that answer fundamental questions in biology and yield advances in regenerative medicine, stem-cell differentiation, and cancer treatment." Anseth will be rec- ognized during the awards ceremony at the 2012 MRS Spring Meeting in San Francisco. The Mid-Career Researcher Award, endowed by Aldrich Materials Science, recognizes exceptional achievements in materials research by mid-career professionals.

Anseth is a leading researcher and inventor in the fields of biomaterials and regenerative medicine. She has shown how control of the chemical, biological, and physical properties of biomaterials enables one to probe fundamental cell biology questions and use this information in targeted applications in tissue regeneration. Her approach is unique in that she combines the ability to synthesize polymers with highly defined structures with an understanding of the molecular dynamics of processes at 